\title{
Correlated disorder in metal-organic frameworks containing asymmetric linkers
}

Emily Maria Reynolds ${ }^{1}$, Alistair Overy ${ }^{1}$, Mia Baise ${ }^{2}$, Ben Slater ${ }^{2}$, Jamie Gould ${ }^{3}$, Andrew Goodwin ${ }^{1}$

${ }^{1}$ University Of Oxford, Oxford, United Kingdom, ${ }^{2}$ University College London, London, United Kingdom, ${ }^{3}$ University of Leeds, Leeds, United Kingdom

E-mail: emily.reynolds@chem.ox.ac.uk

Defects within materials, particularly oxides, are well-known to play an important role in their observed physical and chemical properties. In contrast, the different types of defects and disorder in framework materials are not as well understood, although through the creation of open metal sites and mesopore formation defects are key to accessing properties useful for catalysis and adsorption.[1] Linker and/or metal cluster vacancies have been observed in a variety of metal-organic frameworks (MOFs) including UiO-66, NU-125, MOF-505, and HKUST-1.[1] In these cases, the defective materials exhibit different properties but retain the average structure of the parent material, and as a result, conventional powder diffraction techniques can provide little insight into the location and nature of the defects.

There are very few examples of the long-range 3D order of defects, but like in many disordered systems, short-range correlations often exist. By substituting terephthalate linkers with asymmetric pyrazole-carboxylate linkers into systems such as MOF-5, we introduce disorder into well-known framework materials. The arrangement of these asymmetric linkers within framework materials is a problem analogous to many other systems, either also containing asymmetric linkers, such as cyanide in Prussian blues,[2] or systems which contain atomic displacements such as Nb in KNbO3.[3] These systems allow for the possibilities of both extremes of complete order or disorder, as well as correlated disorder.

We show that while powder and single crystal diffraction data suggest the linkers are disordered, the highly structured diffuse scattering visible in single-crystal X-ray patterns indicates correlated disorder along the linker rows. By comparing experiment with models calculated using simple local rules, we are able to understand the specific type of correlations giving rise to the diffuse, and prove the presence of correlated disorder in a MOF-5 analogue. These results contribute to the ability to identify specific types of correlated disorder within analogous systems, and therefore understand the nature and location of defects.

[1] Fang, Z., Beuken, B, De Vos, D. E. \& Fischer, R. A. (2015). Angewandte Chemie International Edition, 25, $7234-54$.

[2] Karyakin, A. A. (2001). Electroanalysis, 13, 813.

[3] Hewat, A. (1973). Journal of Physics C, 6, 28.

Keywords: MOF, disorder, diffuse 